\title{
Theoretical analysis to interpret projected image data from in-situ 3-dimensional equiaxed nucleation and growth
}

Robin P. Mooney and Shaun McFadden ${ }^{\mathrm{b}, \mathrm{a}}$

aDepartment of Mechanical and Manufacturing Engineering, Trinity College Dublin, Dublin 2, Ireland

${ }^{b}$ Faculty of Computing and Engineering, Ulster University, Magee Campus, Londonderry, Northern Ireland, BT487JL

Corresponding author: Shaun McFadden (s.mcfadden2@ulster.ac.uk)

\begin{abstract}
In-situ observation of crystal growth in transparent media allows us to observe solidification phase change in real-time. These systems are analogous to opaque systems such as metals. The interpretation of transient 2-dimensional area projections from 3-dimensional phase change phenomena occurring in a bulky sample is problematic due to uncertainty of impingement and hidden nucleation events; in stereology this problem is known as overprojection. This manuscript describes and demonstrates a continuous model for nucleation and growth using the well-established Johnson-Mehl-Avrami-Kolmogorov model, and provides a method to relate 3-dimensional volumetric data (nucleation events, volume fraction) to observed data in a 2-dimensional projection (nucleation count, area fraction). A parametric analysis is performed; the over-projection phenomenon is shown to be significant in cases where nucleation is occurring continuously with a relatively large variance. In general, area fraction on a projection plane will overestimate the volume fraction within the sample and the nuclei count recorded on the projection plane will underestimate the number of real nucleation events. The statistical framework given in this manuscript provides a methodology to deal with the differences between the observed (projected) data and the real (volumetric) measures.
\end{abstract}

\section{Keywords:}

A1. Nucleation, A1. Optical Microscopy, A1. Solidification, A2. Microgravity conditions, B1. Alloys, A1. Computer Simulation 


\section{Introduction}

In-situ observation techniques have allowed material scientists to observe phase-change phenomenon in real time [1,2]. Furthermore, the nucleation and growth in some transparent alloy systems are analogous to metallic alloys [3] and are therefore used as model systems. For example, early solidification experiments involved the use of transparent materials (such as $\mathrm{NH}_{4} \mathrm{CL} / \mathrm{H}_{2} \mathrm{O}$ ) [4], where the solidifying phase would exhibit greater opacity than the transparent liquid phase. Hence, solid could be distinguished from liquid in real-time.

More recently, synchrotron x-ray imaging techniques have been applied to the solidification of metallic alloy systems [5-7]. The requirements for in-situ x-ray radiography in metals are that the alloy exhibits partitioning of solute at the solid-liquid interface, and that solute and solvent should have significantly different atomic numbers. If these requirements are fulfilled the process will provide sufficient contrast between the solid and liquid with areas of different compositions observed in the radiographic video sequences.

In-situ observation experiments have provided complimentary data to the traditional post mortem-analyses or ex-situ analysis, however, it is clear that in-situ observation has provided insights previously unobtainable through post-mortem characterisation. For example, dendrite arm fragmentation was first observed in a transparent alloy system [8] and then confirmed in metallic systems using in-situ X-ray radiography [9].

Synchrotron x-ray experiments have provided a significant advancement in the study of alloy solidification for metallurgical purposes; nevertheless, experiments with transparent analogue systems continue to find application in the study of metallurgy. For example, Sturz et al. [10] have recently conducted a solidification experiment on equiaxed dendrites with in-situ observation using the transparent analogue neopentylglycol-camphor on-board the sounding rocket TEXUS-47. Microgravity conditions were achieved on this sounding rocket campaign, hence the crystal growth could be observed in the absence of gravity-driven convection and crystal sedimentation.

It is important to distinguish the dimensionality differences between the transparent systems and the metallic systems for in-situ observation. Metallic systems are necessarily thin (typically less than half a millimetre in thickness) and may be considered as, approximately, 2D specimens in some respects. Whereas transparent samples may have considerable thickness and should be considered as 3D samples. 
If a 3D sample is observed in transmission using one camera then the resulting image is a $2 \mathrm{D}$ projection of a 3D sample. This phenomenon is known as a stereology effect [11], specifically, known as over projection [12]. This manuscript proposes a method to relate observed events in the $2 \mathrm{D}$ projection to actual events in the $3 \mathrm{D}$ sample in real-time during phase change by solidification (polycrystalline nucleation and growth). We assume that the sample is bulky and not a foil, hence, the depth of the sample, $D$, will be greater than the average crystal size. The approach outlined here will have direct application to in-situ observations of transparent systems in microgravity experiments, where the transport effects associated with gravity-driven convection are suppressed [16]. To simplify the analysis, we assume that temperature in the domain is uniform and known a priori.

\section{Method}

Figure 1 shows a system with a transparent initial phase and opaque spherical objects that nucleated from seeds. We assume that the positions of the seeds follow an independent random (Poisson) spatial distribution. Hence, the presence of seed points within the sample occur at any location without bias. If the total number of seeds is given as $n$ then the overall density of seeds is given as $N_{0}=n / V$, where $V$ is the volume of the cuboid. The frontal area of the cuboid is the projection plane and has area, $A$. The depth of the cuboid is $D$.

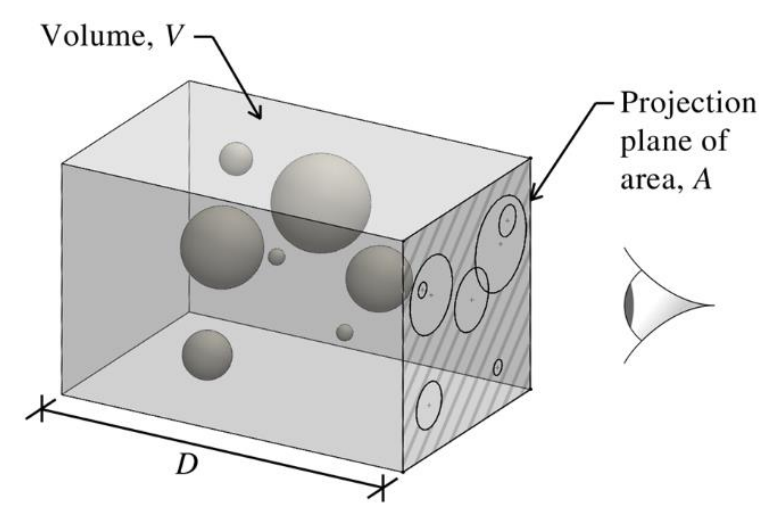

Figure 1 Continuous nucleation and growth of $3 D$ spheres in a cuboid and the corresponding $2 D$ observation on the projection plane.

Spherical growth is assumed at active seed points, hence, under continuous nucleation conditions, and at any point in time, we shall get a distribution of different sphere sizes in 3D space. These spheres may represent a single solid phase or, as is more likely, an envelope containing a mixture of phases (e.g., a mush-containing envelope or a spherule of eutectic). 
The bulk sample is observed from one side only; hence, giving us a 2D projected image of the spherical objects formed. We assume that the depth of field of any magnification instrument is sufficient to allow observation of objects formed at all depths. Two issues arise with the 2D projected image:

1) As the transformation progresses, distinct and separate spherical objects that have not impinged may appear to overlap in the $2 \mathrm{D}$ projected image. This phenomenon gives the impression that adjacent objects have impinged but it is difficult to confirm if the objects have indeed impinged. Figure 1 shows overlapping areas on the projection plane, but it is clear that no spheres within the volume have impinged.

2) Nucleation events may be obstructed from view by larger solid objects. These new nucleation events may have occurred in front of or behind pre-existing solid and will go undetected or uncounted in any nucleation density or rate estimation. Hence, some nucleation events are observed, others are unobserved. This phenomenon has occurred for two spheres in the upper half of Figure 1.

It is interesting to think of the problem from two perspectives. The first perspective is from the experimentalist who is recording the measurable data. Areal data (2D) such as counting of nucleation events and area coverage are measured on the projection plane. But, for posterity, we require volumetric data in $3 \mathrm{D}$. Hence $2 \mathrm{D}$ information must be translated into $3 \mathrm{D}$ data. The second perspective is from the modeller, who is modelling the system in 3D but wishes to compare the model's output with 2D data gathered on the projection plane. Here, the inverse problem applies, where 3D information must be translated into $2 \mathrm{D}$ data. This is the viewpoint taken in this manuscript. Table 1 provides a list of important parameters that may have an areal (2D) interpretation or a volumetric (3D) interpretation.

Table 1

\begin{tabular}{lccc}
\hline Description & Symbol & Unit & Areal or volumetric \\
\hline Total number of potential seed sites & $n$ & {$[-]$} & - \\
Density of all potential seeds & $N_{O}$ & {$\left[\mathrm{~m}^{-3}\right]$} & Volumetric \\
Nucleation density & $N$ & {$\left[\mathrm{~m}^{-3}\right]$} & Volumetric \\
Nucleation rate & $\dot{N}$ & {$\left[\mathrm{~m}^{-3} \mathrm{~s}^{-1}\right]$} & Volumetric \\
Area fraction & $\zeta_{\mathrm{A}}$ & {$[-]$} & Areal \\
Volume fraction & $\zeta_{V}$ & {$[-]$} & Volumetric \\
Nucleation density of real nuclei & $N_{R E A L}$ & {$\left[\mathrm{~m}^{-3}\right]$} & Volumetric \\
Nucleation density of observable nuclei & $N_{O B S}$ & {$\left[\mathrm{~m}^{-2}\right]$} & Areal \\
Observable nucleation events & $n_{O B S}$ & {$[-]$} & Areal \\
\hline
\end{tabular}


The nucleation density at any time is given by $N(t)$ having units of $\left[\mathrm{m}^{-3}\right]$. The nucleation rate, $\dot{N}(t)$, is the time rate of change of the nucleation density given as

$$
\dot{N}(t)=\frac{d N(t)}{d t} .
$$

The nucleation rate has units of $\left[\mathrm{m}^{-3} \mathrm{~s}^{-1}\right]$.

In Classical Nucleation Theory (CNT) the rate of formation of homogeneous nuclei (which is the rate of formation of viable clusters with a critical radius) follows an Arrhenius-type relationship

$$
\dot{N}(t)=C \exp \left(-\frac{\Delta G_{n}}{k_{B} T}\right),
$$

where $C$ is a constant, $\Delta G_{n}$ is the free energy barrier to homogeneous nucleation, $k_{B}$ is the Boltzmann constant, and $T$ is the absolute temperature.

Homogeneous CNT predicts nucleation undercooling values of the order of $100 \mathrm{~K}$, which are impractical and rarely realised in the majority of solidification cases. Instead heterogeneous nucleation, which occurs on pre-existing substrate surfaces such as container walls or on particles/inclusions within the melt, is expected. In heterogeneous CNT the wetting conditions on the substrate surface are taken into account; hence, the free-energy barrier to nucleation is reduced by a factor $f(\theta)$, where $0<f(\theta)<1$ and the heterogeneous nucleation rate has the form

$$
\dot{N}(t)=C \exp \left(-\frac{\Delta G_{n}}{k_{B} T} f(\theta)\right) .
$$

Heterogeneous CNT leads to lower nucleation undercooling values (typically, <15 K). An alternative to CNT is to consider thermally activated nucleation conditions as a continuous distribution on preferential nucleation sites, described by $d N / d(\Delta T)$. The undercooling is $\Delta T=T_{L}-T$ and $T_{L}$ is the equilibrium liquidus temperature.

The nucleation rate can be determined if the cooling rate, $\dot{T}$, is known, that is,

$$
\dot{N}(t)=\frac{d N}{d(\Delta T)} \frac{d\left(T_{L}-T\right)}{d t}=-\dot{T} \frac{d N}{d(\Delta T)},
$$

In 1966, Oldfield [14] was the first to propose an athermal distribution law for $d N / d(\Delta T)$. In 1989, Rappaz [15] reviewed the use of standard statistical distributions to describe the nucleation conditions, where generally 


$$
\frac{d N}{d(\Delta T)}=N_{o} p(\Delta T)
$$

$N_{o}$ is the volumetric density of all potential nucleation sites and $p(\Delta T)$ is a probability density function. A Gaussian distribution probability density function, $p_{\text {Gauss. }}(\Delta T)$, may be applied as follows

$$
\frac{d N}{d(\Delta T)}=N_{o} p_{\text {Gauss. }}(\Delta T)=\frac{N_{o}}{\Delta T_{\sigma} \sqrt{2 \pi}} \exp \left[-\frac{1}{2}\left(\frac{\Delta T-\Delta T_{o}}{\Delta T_{\sigma}}\right)^{2}\right] .
$$

Here $\Delta T_{o}$ is the overall average nucleation undercooling and $\Delta T_{\sigma}$ is the standard deviation of the undercooling distribution.

The use of statistical distributions to describe nucleation conditions was lacking credible physical explanation until, in 2000, Greer and co-workers [13] provided much-needed insight. They showed that for potent nucleating particles, the heterogeneous nucleation stage itself was not the controlling factor. The free-energy barrier to heterogeneous nucleation is reduced on a substrate particle surface, but there is a further barrier to free-growth of solid due to the finite geometry of the substrate particle. This free growth barrier depends on particle size. Free growth on a particle occurs at the undercooling value where the nucleated solid (described by the heterogeneous cap model) reaches hemi-spherical proportions on that particle. It was shown that the undercooling required for free-growth is inversely proportional to the particle size. Greer et al. argued that particle sizes should follow a distribution and, therefore, initiation undercooling values will also follow a distribution. In their case, a lognormal distribution of particle sizes was assumed for convenience, thus leading to an athermal grain nucleation theory described by a statistical distribution on undercooling. The suitability of using statistical distribution to describe nucleation conditions is wellestablished, and indeed, the CNT is largely redundant in the specialised field of grain refinement.

Our analysis proceeds on the basis of application of a Gaussian distribution to determine the nucleation conditions. We assume that the free-growth or initiation stage defines the nucleation of an active seed. Hence, the nucleation rate is given as

$$
\dot{N}(t)=\frac{-\dot{T} N_{o}}{\Delta T_{\sigma} \sqrt{2 \pi}} \exp \left[-\frac{1}{2}\left(\frac{\left(T_{L}-T\right)-\Delta T_{o}}{\Delta T_{\sigma}}\right)^{2}\right] .
$$

At any given time, $t$, the density of active nuclei, $N_{A C T I V E}$, is obtained by integrating with respect to nucleation time, $t_{n}$, where 


$$
N_{\text {ACTIVE }}(t)=\int_{0}^{t} \dot{N}\left(t_{n}\right) d t_{n} .
$$

The nucleation time variable is an independent time variable that is required for integration purposes. The number of active nuclei may be estimated (assuming uniform conditions) by

$$
n_{\text {ACTIVE }}(t)=\int_{V} N_{A C T I V E} d V=V N_{A C T I V E} .
$$

At any given time, the remaining inactive seed particles are passive because they have not reached their nucleation temperature. The passive seeds will have a density given by

$$
N_{\text {PASSIVE }}(t)=N_{o}-N_{\text {ACTIVE }}(t) \quad .
$$

Because we assume a Poisson spatial distribution, we should realise that not all seed nucleation events (active seeds) are real events. Strictly, the Poisson distribution will permit phantom nucleation. Phantom nucleation is described as the nucleation of a seed occurring within an already transformed volume (i.e., engulfment of seeds), hence we do not physically observe the phantom nucleation event . The Johnson-Mehl-Avrami-Kolmogorov (JMAK) approach is an established method based on Poisson statistics and it is derived by accounting for phantom growth and impingement during a change of state (reviewed in $[16,17])$. It is well-known that phantom nucleation is a strict mathematical requirement for the JMAK equation given as

$$
\zeta_{V}=1-\exp \left(-\zeta_{V, E X}\right)
$$

Where $\zeta_{V}$ is the volume fraction and is defined as the union of all discrete transformed volumes, $V_{i}$, divided by the total volume, $V$,

$$
\zeta_{V}=\frac{\bigcup_{i=1}^{n} V_{i}}{V}
$$

On the other hand, $\zeta_{V, E X}$ is known as the extended volume fraction and may be calculated two ways as follows,

$$
\zeta_{V, E X}=\frac{\sum_{i=1}^{n} V_{i}}{V}=\sum_{i=1}^{n} N_{i} V_{i}
$$

The term $N_{i}$ is the nucleation density at the time of nucleation: $t_{n i}$ (of any given seed $i$ ). In the continuum limit, the extended volume is given as

$$
\zeta_{V, E X}(t)=\frac{4 \pi}{3} \int_{0}^{t} \dot{N}\left(t_{n}\right)\left[\int_{t_{n}}^{t} v_{t i p} d t^{\prime}\right]^{3} d t_{n}
$$


where $v_{\text {tip }}$ is the growth velocity and time variable $t$ ' is another intermediate integration time variable.

Kolmogorov is accredited with the application of Poisson statistics for the derivation of the JMAK equation (cited in [17]). The probability that a nucleation event is real (i.e., that it occurred in an untransformed region) is given by $P_{R E A L}=\left(1-\zeta_{V}\right)$ and the nucleation rate for real nuclei is expanded as follows, $\dot{N}_{R E A L}(t)=\dot{N}(t) \cdot\left(1-\zeta_{V}\right)-N(t) \cdot \dot{\zeta}_{V}$. The first term on the right side in this equation represents the nucleation rate of independent real nucleation events occurring in untransformed regions. The second term represents a reduction rate due to previously nucleated real events that are subsequently consumed by the growth of the transforming region (a transfer rate of real nuclei to phantom nuclei). In our framework, we are only concerned with the establishment of real nuclei on the basis that real nuclei should remain real. Hence, we ignore the second term to get $\dot{N}_{R E A L}(t)=\dot{N}(t) \cdot\left(1-\zeta_{V}\right)$ and, over time, the nucleation density of real nuclei is obtained by integrating the first term only

$$
N_{R E A L}(t)=\int_{0}^{t} \dot{N}\left(t_{n}\right) \cdot\left(1-\zeta_{V}\right) d t_{n} .
$$

The probability of a nucleation event being phantom is $P_{P H}=\zeta_{V}$ and the expanded rate equation for phantom nucleation is $\dot{N}_{P H}(t)=\dot{N}(t) \cdot \zeta_{V}+N(t) \cdot \dot{\zeta}_{V}$. The first term represents the nucleation rate of phantom nuclei occurring within previously transformed regions. The second term is the transfer rate of real nuclei to phantom nuclei due to growth of the transforming region. Following a previous argument, we ignore the second term to give $\dot{N}_{P H}(t)=\dot{N}(t) \cdot \zeta_{V}$, and the nucleation density of phantom nuclei is calculated as

$$
N_{P H}(t)=\int_{0}^{t} \dot{N}\left(t_{n}\right) \cdot \zeta_{V} d t_{n} .
$$

The number of real and phantom nucleation events, respectively, are obtained as follows

$$
\begin{aligned}
n_{R E A L}(t) & =N_{R E A L}(t) V, \\
n_{P H}(t) & =N_{P H}(t) V .
\end{aligned}
$$

Similarly, in $2 \mathrm{D}$, the area fraction, $\zeta_{A}$, on the projected plane is defined as

$$
\zeta_{A}=\frac{\bigcup_{i=1}^{n} A_{i}}{A}
$$

Which is the union of all projected areas, $A_{i}$, divided by the area of the projection plane.

If the Poisson assumption holds, the 2D form of the JMAK equation is given as

$$
\zeta_{A}=1-\exp \left(-\zeta_{A, E X}\right)
$$


where $\zeta_{A, E X}$ is known as the extended area. Extended area is defined as

$$
\zeta_{A, E X}=\frac{\sum_{i=1}^{n} A_{i}}{A}=\sum_{i=1}^{n} N_{i} D A_{i} .
$$

Multiplying the nucleation density $N_{i}$ by the depth $D$ gives us a volumetric to areal transformation (from nuclei per unit volume $\left[\mathrm{m}^{-3}\right]$ to nuclei per unit area $\left[\mathrm{m}^{-2}\right]$ ).

In the continuum limit, the extended area fraction is given by

$$
\zeta_{A, E X}(t)=\pi D \int_{0}^{t} \dot{N}\left(t_{n}\right)\left[\int_{t_{n}}^{t} v_{t i p} d t^{\prime}\right]^{2} d t_{n}
$$

Using a similar argument to the previous volumetric case, the JMAK equation is used to provide the probability that a nucleation event may be observed on the projection plane. At any given time, the probability that a nucleation event is observable is $P_{O B S}=\left(1-\zeta_{A}\right)$. The nucleation density of observable nuclei is calculated as

$$
N_{O B S}(t)=\int_{0}^{t} \dot{N}\left(t_{n}\right) \cdot D \cdot\left(1-\zeta_{A}\right) d t_{n}
$$

We should recall that not all active nucleation events are real, because some are phantom, and not all real nucleation events are observable. Recognising the relationship: $\dot{N}(t)=$ $\dot{N}_{R E A L}(t) /\left(1-\zeta_{V}\right)$, we can establish the relationship between observable nucleation events (in 2D) and real nucleation events (in 3D).

$$
N_{O B S}(t)=\int_{0}^{t} \dot{N}_{R E A L}\left(t_{n}\right) \cdot D \cdot \frac{\left(1-\zeta_{A}\right)}{\left(1-\zeta_{V}\right)} d t_{n}
$$

We can give meaning to each of the terms in the integrand of equation (24). $\dot{N}_{R E A L}(t)$ is the nucleation rate of real nuclei. The depth, $D$, provides a volumetric (3D) to areal (2D) transformation. The term $\left(1-\zeta_{A}\right)$ is the probability that a nucleation event will be observed in $2 \mathrm{D}$. The term $\left(1-\zeta_{V}\right)$ is a correction factor that allows us to use Poisson statistics in the analysis. This correction factor is required because real nucleation events can only take place in untransformed volume and are, by definition, excluded from the previously transformed volume. The application of this correction factor restores randomness to the entire volume and hence to the projected area. The application of the correction factor is found elsewhere in literature, for example, Tomellini and Fanfoni [18] discuss its application for mixed mode nucleation of thin films onto solid surfaces. 
Finally, the number of observable nuclei on the $2 \mathrm{D}$ projected plane is given by

$$
n_{O B S}(t)=N_{O B S}(t) A
$$

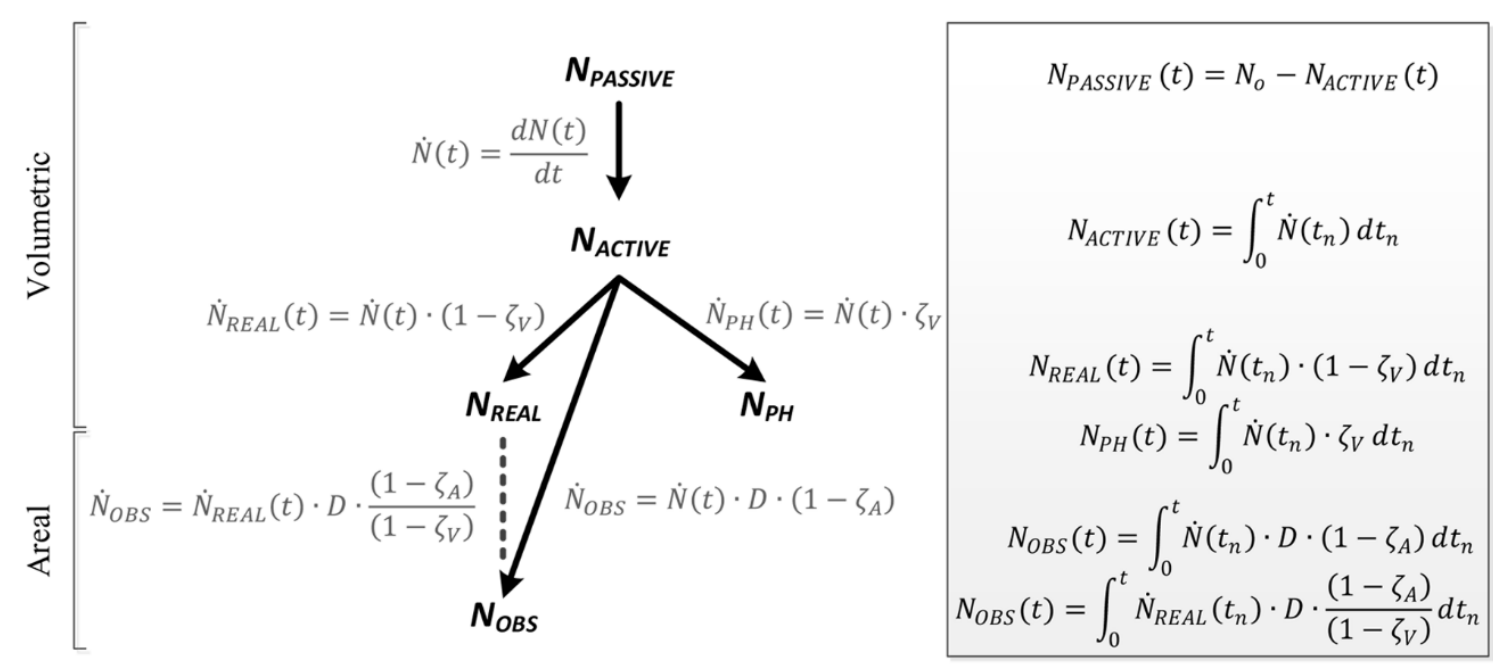

Figure 2: Flowchart for the calculation of nucleation densities.

Figure 2 shows a flowchart highlighting the mathematical operations required to determine the nucleation densities. The rate equations are shown and the integral equations are listed on the right hand side. There are two options to determine the nucleation density $N_{O B S}$ : use equation (23) or equation (24).

The terminology used to distinguish between Passive and Active seeds; Real and Phantom nucleation; and Observed nucleation events is deemed to be a key aspect of this proposed model.

\subsection{Summarising measures}

In the limit, the final density of real nucleation events will be less than $N_{o}$, hence we may define the nucleation efficiency as the fraction of all real nucleation events to the number of possible nucleation events. The nucleation efficiency is given by

$$
\eta_{R E A L}=\lim _{t \rightarrow \infty} \frac{N_{R E A L}(t)}{N_{o}}
$$

Furthermore, due to viewing obstruction, the number of observed nucleation events will be less than or equal to the number of real nucleation events. Hence, we may define the observed nucleation efficiency as

$$
\eta_{O B S}=\lim _{t \rightarrow \infty} \frac{N_{O B S}(t)}{D N_{O}}
$$


Another consideration is the reduction in average nucleation undercooling values: the real average undercooling and the observed average undercooling.

We require a general method to determine the mean value of undercooling for all real and observed nucleation events. In statistics, the 'expected value' equation provides a method to calculate the mean undercooling, $\overline{\Delta T}$, from a known continuous distribution of nucleation events, as follows

$$
\overline{\Delta T}=\int_{0}^{\infty} p(\Delta T) \cdot \Delta T d(\Delta T)
$$

where $p(\Delta T)$ is the probability density function (PDF) of the known distribution. (In our case, the known distribution is that output by the model, i.e., the nucleation distribution of real or observed nucleation events.) We assume that the integration limits range from zero to infinity with negligible consequences.

The PDF is given by the product of a normalising constant, $\varphi$, and the distribution of nucleation density (the simulation output) on nucleation undercooling, $N_{\text {output }} / d(\Delta T)$, as follows

$$
p(\Delta T)=\varphi \frac{d N_{\text {output }}}{d(\Delta T)} .
$$

The normalising constant is selected to ensure that the integral of $p(\Delta T)$ between the limits is unity; this is a strict requirement of a PDF [19] and equation (28). Specific PDFs can be formulated by selecting the appropriate nucleation density output (either $N_{R E A L}$ or $N_{O B S}$ ) and its normalising constant.

Recognising that under constant cooling conditions

$$
d(\Delta T)=-\dot{T} d t
$$

and with a starting condition of $\Delta T=0$ at $t=0$,

$$
\Delta T=-\dot{T} t .
$$

Substituting equations (29), (30) and (31) into equation (28) gives a general equation for average nucleation undercooling in a known distribution of nucleation density, as follows

$$
\overline{\Delta T}=-\varphi \dot{T} \int_{0}^{\infty}-\dot{T} \frac{d N_{\text {output }}}{d(\Delta T)} t d t=-\varphi \dot{T} \int_{0}^{\infty} \dot{N}_{\text {output }}(t) t d t
$$

Hence, with specific application to the real nucleation rate, the real average undercooling is given by 


$$
\overline{\Delta T}_{R E A L}=\frac{-\dot{T}}{\lim _{t \rightarrow \infty} N_{R E A L}(t)} \int_{0}^{\infty} t \dot{N}_{R E A L} d t
$$

and the normalisation constant is $\varphi=1 /\left[\lim _{t \rightarrow \infty} N_{R E A L}(t)\right]$.

The observed average undercooling is given by

$$
\overline{\Delta T}_{O B S}=\frac{-\dot{T}}{\lim _{t \rightarrow \infty} N_{O B S}(t)} \int_{0}^{\infty} t \dot{N}_{O B S} d t,
$$

and the normalisation constant is $\varphi=1 /\left[\lim _{t \rightarrow \infty} N_{O B S}(t)\right]$.

Both the real and observed average undercooling values must be less than or equal to the overall average nucleation undercooling, $\Delta T_{o}$ (a simulation input, defined in equation (6)). Hence, it is useful to define the following ratios for average nucleation undercooling values (real and observed) against the overall average of the full Gaussian distribution, as follows

$$
R_{R E A L}=\frac{\overline{\Delta T}_{R E A L}}{\Delta T_{o}},
$$

and

$$
R_{O B S}=\frac{\overline{\Delta T}_{O B S}}{\Delta T_{o}}
$$

\section{Results and discussion}

The model is demonstrated by assuming a cuboid volume with the following dimensions: Height, $H=10 \mathrm{~mm}$; Width, $W=13 \mathrm{~mm}$; and Depth, $D=3 \mathrm{~mm}$. The growth rate is assumed to follow a growth law, $v_{t i p}=C \Delta T^{b}$, where $C=6.544 \times 10^{-10} \mathrm{~m} / \mathrm{s} /{ }^{\circ} \mathrm{C}^{4}$ and $b=4$. A baseline simulation case is provided initially to demonstrate a typical simulation output. The baseline nucleation parameters are $N_{\mathrm{o}}=1 \times 10^{10} / \mathrm{m}^{3}, \Delta T_{0}=8{ }^{\circ} \mathrm{C}$, and $\Delta T_{\sigma}=1{ }^{\circ} \mathrm{C}$. Furthermore, the cooling rate is set to a baseline value of $\dot{T}=0.75^{\circ} \mathrm{C} / \mathrm{min}$.

These simulation inputs are demonstrative. It is noted, however, that the cuboid volume dimensions, growth law settings, cooling rate, overall average nucleation undercooling, and standard deviation of nucleation undercooling are typical of that used, and observed in, a recent microgravity equiaxed crystal growth experiment involving the transparent analogue alloy neopentylglycol-camphor [20]. 


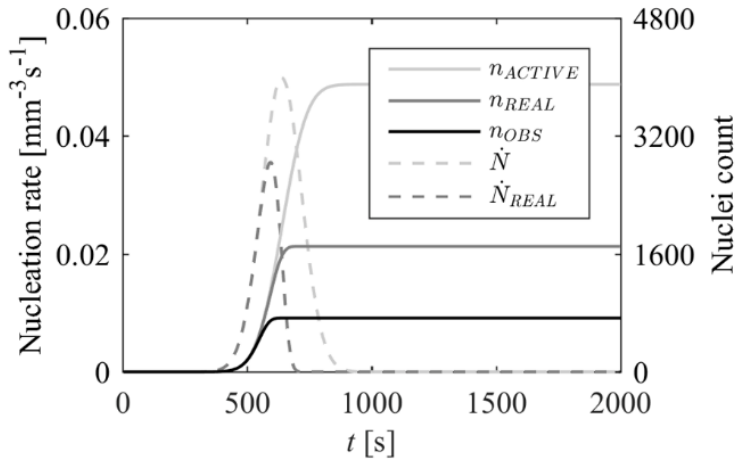

Figure 3 Simulation output for the baseline case: nucleation rates and nuclei counts versus time

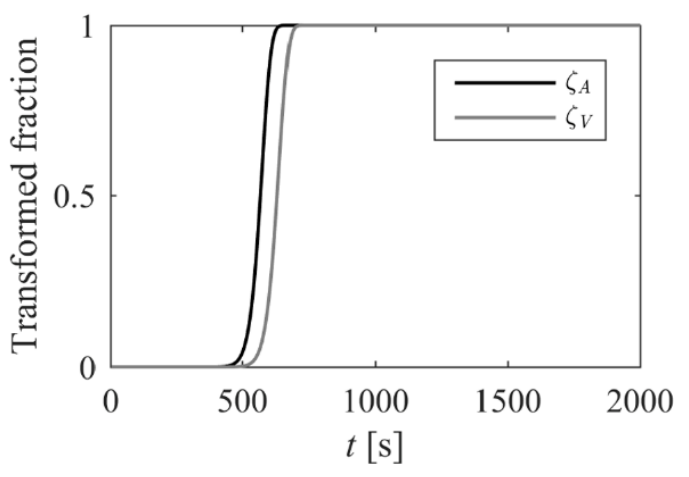

Figure 4 Simulation output for the baseline case: area fraction and volume fraction versus time.

Figure 3 shows the overall nucleation rate, $\dot{N}$, and the number of active nuclei, $n_{A C T I V E}$ (which is a cumulative function). The number of active nuclei saturated at approximately at 3900 nuclei. This value corresponds to the total number of potential seeds in the volume - under the Poisson assumption, all nuclei become active with time. However, due to the increase in volume fraction, the real nucleation rate, $\dot{N}_{R E A L}$, was lower than expected and peaked at an earlier time than the overall nucleation rate. Hence, the number of real nuclei, $n_{R E A L}$, was lower than the number of active nuclei. The numerical difference between these two nuclei counts at any time would give the number of phantom nuclei. Finally, the number of observed nucleation events on the projected plane, $n_{O B S}$, was predicted to be lower than the number of real nucleation events. This reduction was due to the stereology effect as described by either equation (23) or (24).

Figure 4 shows the corresponding area fraction and volume fraction for the baseline simulation case. It is clear that area fraction is greater than volume fraction throughout the process of nucleation.

To demonstrate the model further, a parametric analysis is presented where the cooling rate and the average overall undercooling are varied. A range of cooling rates $\left(\dot{T}=0.375{ }^{\circ} \mathrm{C} / \mathrm{min}\right.$, $0.75{ }^{\circ} \mathrm{C} / \mathrm{min}$, and $\left.1.5^{\circ} \mathrm{C} / \mathrm{min}\right)$ and average nucleation undercooling values $\left(\Delta T_{0}=4{ }^{\circ} \mathrm{C}, 8{ }^{\circ} \mathrm{C}\right.$, and $12{ }^{\circ} \mathrm{C}$ ), were simulated. The standard deviation of nucleation undercooling was fixed at $\Delta T_{\sigma}=1{ }^{\circ} \mathrm{C}$; the seed density in the volume also remained fixed at $N_{\mathrm{o}}=1 \times 10^{10} / \mathrm{m}^{3}$.

Figure 5 shows a matrix of plots obtained during the parametric study of the model. The cooling rate increases from top to bottom of the matrix and the nucleation undercooling increases from left to right. Each plot, labelled (a) to (i), shows the nucleation rate on the primary $y$-axis, the number of nuclei on the secondary $y$-axis, and the simulation time on the 
$x$-axis. Additional output data for each simulation case is presented above each plot. The nucleation efficiency $\left(\eta_{R E A L}\right)$, the observed nucleation efficiency $\left(\eta_{O B S}\right)$, and the ratios of average undercooling levels $\left(R_{R E A L}\right.$ and $\left.R_{O B S}\right)$ are provided.


$\dot{N}---\dot{N}_{R E A L}$

$n_{A C T I V E}$

$n_{R E A L}$

$n_{O B S}$

Figure 5 Simulation outputs from the parametric analysis.

The central plot, Figure 5 (e), is the baseline simulation case that was previously discussed. Some general trends from the parametric analysis can be identified. As cooling rate $(\dot{T})$ was increased, the nucleation rate tended to increase and peak at earlier time values. Additionally, the nucleation efficiency tended to increase with cooling rate. This behaviour is expected since at higher cooling rates we expect more particles to nucleate in any given time frame compared to that at a lower cooling rate. A short nucleation time frame leads to less 
engulfment and therefore higher nucleation efficiency. In the limit, as cooling rate increases we asymptotically approach big-bang nucleation, where all seeds nucleate at once, i.e., 100\% nucleation efficiency.

As the overall average undercooling $\left(\Delta T_{\mathrm{o}}\right)$ increased, nucleation tended to occur later in the simulation; and nucleation efficiencies dropped significantly. This behaviour is due to the power law form of the growth law (a function of undercooling to the fourth power). High undercooling values give more opportunity for engulfment, since the first seeds to nucleate grow rapidly and occupy the majority of the bulk volume, therefore giving lower nucleation efficiencies. The reduction in $R_{R E A L}$ and $R_{O B S}$ is also explained by this effect since the first seeds to nucleate will be those having the lowest nucleation undercooling values within the Gaussian distribution.

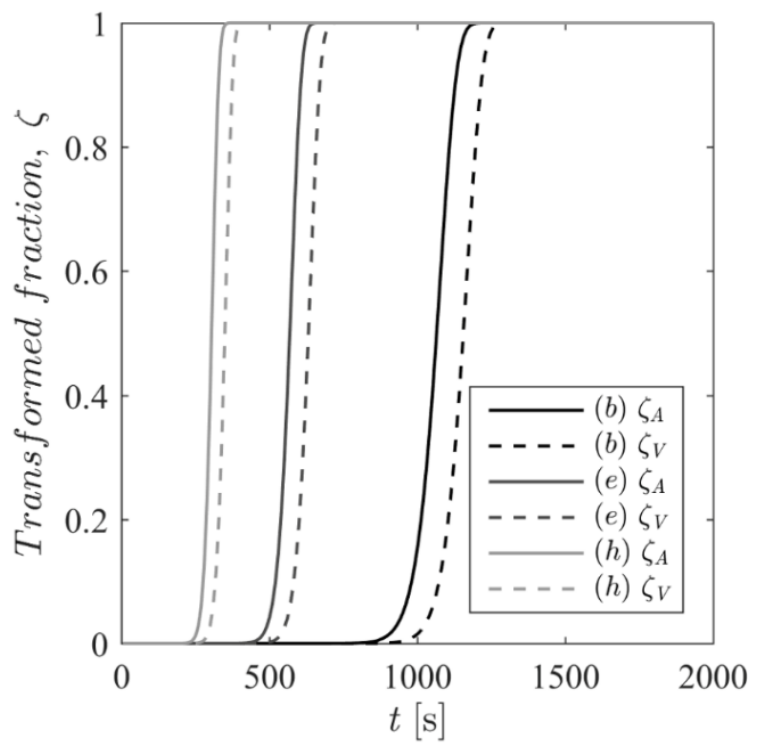

Figure 6 Transformed fraction (areal and volumetric) versus time.

Figure 6 shows the simulated area fractions, $\zeta_{A}$, and volume fractions, $\zeta_{V}$, for simulation cases (b), (e), and (h) (corresponding to the central column of plots in Figure 5 where $\Delta T_{\mathrm{o}}=8$ $\left.{ }^{\circ} \mathrm{C}\right)$. In each case, the area fraction increases ahead of the volume fraction, which is due to over-projection in the $2 \mathrm{D}$ projection plane before impingement events occur within the volume. Considering again Figure 5 (e); the final value of the observable nucleation events was $n_{O B S}=700$. This value is significantly lower than the final number of real nucleation events estimated to have taken place $\left(n_{R E A L}=1700\right)$. 
Figure 7 shows a plot of real and observed nucleation efficiencies plotted against cooling rate at the three levels of overall average nucleation undercooling. Generally, increasing the cooling rate increases nucleation efficiency. At the lower value of average nucleation undercooling $\left({ }^{\circ} \mathrm{C}\right)$ the nucleation efficiencies were highest at all cooling rates. Given the power law form of the growth law (mentioned above), the growth rate is expected to have a stronger effect at the higher levels of undercooling. In the cases where high undercooling values were reached, real nucleation rates were lowered significantly. Fast growth effects (high $\left.\zeta_{V}\right)$ dominated the real nucleation rate equation, $\dot{N}_{R E A L}=\dot{N}\left(1-\zeta_{V}\right)$.

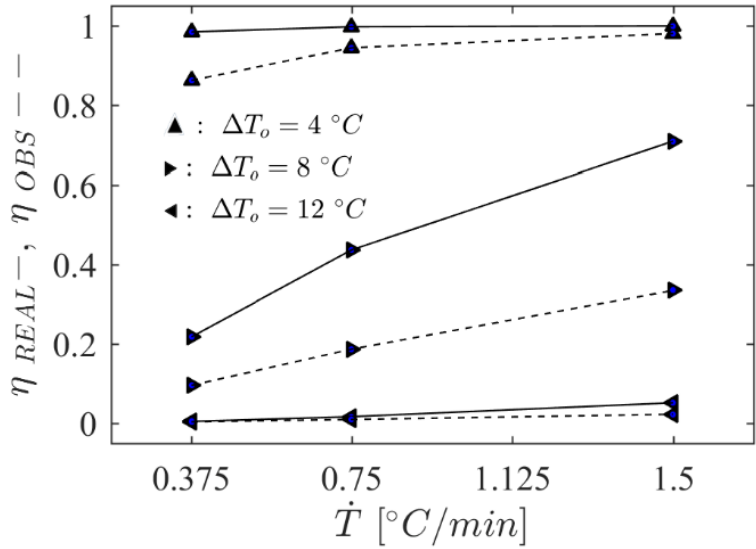

Figure 7 Real and observed nucleation efficiency versus cooling rate. (Real, solid lines; Observed, broken lines)

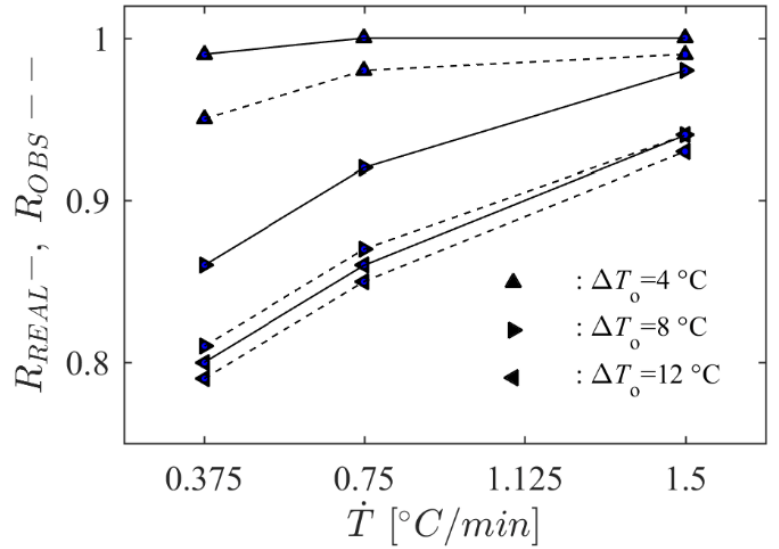

Figure 8 Real and observed ratios of average nucleation undercooling versus cooling rate. (Real, solid lines; Observed, broken lines)

Figure 8 shows how the ratio of average undercooling levels changes for each simulation. It is clear that in cases with low nucleation efficiencies, the real and observed average undercooling levels are less than the overall average.

Figures 7 and 8 also demonstrate the stereology effects on the results. The stereology effect is significant where nucleation is allowed to occur continuously over a finite time frame, for example, at the mid-level average nucleation undercooling $\left(8^{\circ} \mathrm{C}\right)$ in both figures.

\section{Conclusion}

In conclusion, when nucleation and growth in the bulk sample is observed in-situ and from one side (on a 2D projection plane), and if nucleation is continuous, then area fraction on the observed plane will overestimate the volume fraction within the volume (due to the stereological effect of over-projection). And, in the latter stages, nucleation and growth 
events may be blocked from view, thus providing an underestimate of the number of real nucleation events. A statistical approach to determine the relationships between the volumetric data in the bulk sample and the areal data observed on a projection plane has been outlined. The theory is presented in detail and demonstrated using realistic input data. Insight into the stereological effect and the relationship with cooling rate and nucleation undercooling is achieved using a parametric analysis. The stereological effect is seen to be significant for several realistic cases of continuous distributions with reasonable values of cooling rate. This theoretical analysis will find application in the observation of transparent systems, for example, in microgravity solidification experiments.

\section{Acknowledgement}

The authors would like formally acknowledge L. Sturz and G. Zimmermann of ACCESS e.V. for useful discussions and for providing typical geometry and crystal growth data. This work has been carried out as part of the CETSOL (Columnar-to-Equiaxed Transition in Solidification Processing) European Space Agency microgravity application programme. The authors would like to acknowledge the financial support of the ESA PRODEX Programme (contract number 4000107132) and the managerial support of the Irish Delegation to ESA within Enterprise Ireland.

\section{References}

[1] S. Akamatsu, H. Nguyen-Thi, In situ observation of solidification patterns in diffusive conditions, Acta Mater. 108 (2016) 325-346. doi:10.1016/j.actamat.2016.01.024.

[2] V.T. Witusiewicz, U. Hecht, S. Rex, Top-view approach for in-situ observation of growth morphology in bulk transparent organic alloys, J. Cryst. Growth. 353 (2012) 17-24. doi:10.1016/j.jcrysgro.2012.04.040.

[3] K.A. Jackson, J.D. Hunt, Transparent compounds that freeze like metals, Acta Metall. 13 (1965) 1212-1215. doi:10.1016/0001-6160(65)90061-1.

[4] M.H. Burden, J.D. Hunt, Some observations on primary dendrite spacings, Met. Sci. 10 (1976). doi:10.1179/030634576790431886.

[5] R. Mathiesen, L. Arnberg, F. Mo, T. Weitkamp, A. Snigirev, Time Resolved X-Ray Imaging of Dendritic Growth in Binary Alloys, Phys. Rev. Lett. 83 (1999) 5062-5065. doi:10.1103/PhysRevLett.83.5062. 
[6] R.H. Mathiesen, L. Arnberg, K. Ramsøskar, T. Weitkamp, C. Rau, A. Snigirev, Timeresolved x-ray imaging of aluminum alloy solidification processes, Metall. Mater. Trans. B. 33 (2002) 613-623. doi:10.1007/s11663-002-0041-2.

[7] A. Prasad, S.D. McDonald, H. Yasuda, K. Nogita, D.H. StJohn, A real-time synchrotron X-ray study of primary phase nucleation and formation in hypoeutectic Al-Si alloys, J. Cryst. Growth. 430 (2015) 122-137. doi:10.1016/j.jcrysgro.2015.06.024.

[8] K.A. Jackson, J.D. Hunt, D.R. Uhlmann, T.P. Seward, On the origin of the equiaxed zone in castings, Trans. Metall. Soc. AIME. 236 (1966) 151-158. http://www.scopus.com/inward/record.url?eid=2-s2.00001577492\&partnerID=tZOtx3y1.

[9] R.H. Mathiesen, L. Arnberg, P. Bleuet, A. Somogyi, Crystal fragmentation and columnar-to-equiaxed transitions in Al-Cu studied by Synchrotron $\mathrm{X}$-ray video microscopy, Metall. Mater. Trans. A Phys. Metall. Mater. Sci. 37 (2006) 2515-2524. doi:10.1007/BF02586224.

[10] L. Sturz, G. Zimmermann, In-situ and real-time investigation of the columnarequiaxed transition in the transparent alloy system neopentylglycol-camphor onboard the sounding rocket TEXUS-47, J. Phys. Conf. Ser. 327 (2011). doi:10.1088/1742$6596 / 327 / 1 / 012002$.

[11] P. Davy, R.E. Miles, Sampling Theory for Opaque Spatial Specimens, J. R. Stat. Soc. Ser. B. 39 (1977) 56-65. http://www.jstor.org/stable/2984879.

[12] E.E. Underwood, Stereology, or the quantitative evaluation of microstructures, J. Microsc. 89 (1969) 161-180. doi:10.1111/j.1365-2818.1969.tb00663.x.

[13] A. Greer, A. Bunn, A. Tronche, P. Evans, D. Bristow, Modelling of inoculation of metallic melts: application to grain refinement of aluminium by $\mathrm{Al}-\mathrm{Ti}-\mathrm{B}, \mathrm{Acta}$ Mater. 48 (2000) 2823-2835. doi:10.1016/S1359-6454(00)00094-X.

[14] W. Oldfield, A quantitative approach to casting solidification: freezing of cast iron, Trans. ASME. 59 (1966) 945-961.

[15] M. Rappaz, Modelling of microstructure formation in solidification processes, Int. Mater. Rev. (1989). doi:10.1179/imr.1989.34.1.93.

[16] F. Liu, F. Sommer, C. Bos, E.J. Mittemeijer, Analysis of solid state phase 
transformation kinetics: models and recipes, Int. Mater. Rev. 52 (2007) 193-212. doi:10.1179/174328007X160308.

[17] M. Fanfoni, M. Tomellini, The Johnson-Mehl-Avrami-Kolmogorov model: A brief review, Nuovo Cim. D. 20 (1998) 1171-1182. doi:10.1007/BF03185527.

[18] M. Tomellini, M. Fanfoni, Why phantom nuclei must be considered in the JohnsonMehl-Avrami-Kolmogoroff kinetics, Phys. Rev. B. 55 (1997) 14071-14073. doi:10.1103/PhysRevB.55.14071.

[19] N.S. Matloff, Probability modelling and computer simulation, PWS-Kent, Boston, MA, 1998.

[20] L. Sturz, M. Hamacher, G. Zimmermann, In-situ observation of equiaxed dendritic growth and interaction in microgravity, in: Z. Fan (Ed.), 6th Decenn. Int. Conf. Solidif. Process., 2017: pp. 300-303. 